\title{
Exploring Timelike Region for the Meson-Photon Transitions in the Light-Front Quark Model
}

\author{
Ho-Meoyng Choi*† \\ Department of Physics Education, Kyungpook National University, Daegu 41566, Korea \\ E-mail: homyoung@knu.ac.edu
}

\section{Hui-Young Ryu}

Department of Physics, Pusan National University, Pusan 46241, Korea

E-mail: ryulab2012@gmail.com

\section{Chueng-Ryong Ji}

Department of Physics, North Carolina State University, Raleigh, North Carolina 27695-8202,

USA

E-mail: crjiencsu.edu

\begin{abstract}
We investigate the $\left(\pi^{0}, \eta, \eta^{\prime}\right) \rightarrow \gamma^{*} \gamma$ transitions both for the spacelike region and the timelike region using the light-front quark model (LFQM). In particular, we present the new direct method to explore the timelike region without resorting to mere analytic continuation from the spacelike region to the timelike region. Our direct calculation in timelike region shows the complete agreement not only with the analytic continuation result from the spacelike region but also with the result from the dispersion relation between the real and imaginary parts of the form factor.
\end{abstract}

XVII International Conference on Hadron Spectroscopy and Structure - Hadron2017

25-29 September, 2017

University of Salamanca, Salamanca, Spain

\footnotetext{
*Speaker.

${ }^{\dagger}$ HMC was supported by Kyungpook National University Research Fund, 2017. HYR was supported by the NRF grant funded by the Korea government(MSIP) (No. 2015R1A2A2A01004238). CRJ was supported in part by the US Department of Energy (Grant No. DE-FG02-03ER41260).
} 


\section{Introduction}

The meson-photon transitions have been known to be the simplest exclusive processes involving the strong interaction and play a significant role in allowing both the low- and high-energy precision tests of the standard model. For the low-energy regime, the transition form factors (TFFs) enter the prediction of important observables such as the hadronic light-by-light scattering contribution to the muon anomalous magnetic moment [1]. For the high-energy regime, the TFFs can be calculated asymptotically at leading twist as a convolution of the perturbative hard scattering amplitude and the gauge-invariant meson distribution amplitude (DA) which incorporates the nonperturbative dynamics of QCD bound state. The pQCD prediction for $F_{\pi \gamma}\left(Q^{2}\right)$ is given by $Q^{2} F_{\pi \gamma}\left(Q^{2} \rightarrow \infty\right)=\sqrt{2} f_{\pi}$ [2]. However, the measurements of the BaBar Collaboration [3] have shown not only the serious violation of the $\mathrm{pQCD}$ limit but also the rapid growth for $Q^{2}>15 \mathrm{GeV}^{2}$. On the other hand, the measurements from the Belle Collaboration [4] have shown consistency with the asymptotic limit of QCD for $Q^{2}>15 \mathrm{GeV}^{2}$. These discrepancies between the BaBar and the Belle data have motivated many theoretical studies to understand and reconcile those discrepancies.

To examine the issue of the scaling behavior of $Q^{2} F_{\pi \gamma}\left(Q^{2}\right)$ in the large $Q^{2}$, it seems necessary to analyze the TFF not only in the spacelike region but also in the timelike region. Thus, we attempt to explore the entire timelike region as well as the spacelike region in this work. We extend our previous analysis [6] for the single-virtual $P \rightarrow \gamma^{*} \gamma\left(P=\pi^{0}, \eta, \eta^{\prime}\right)$ transition in the spacelike region using the light-front quark model (LFQM) [7] to include the entire timelike region.

\section{Model Description}

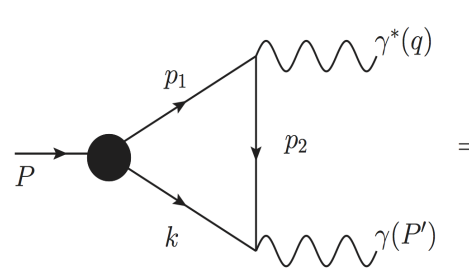

(a)

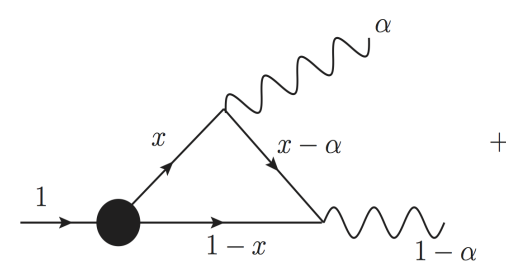

(b)

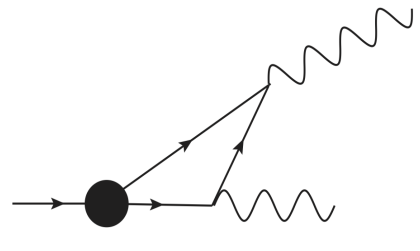

(c)

Figure 1: One-loop Feynman diagrams that contribute to $P \rightarrow \gamma^{*} \gamma$. The single covariant Feynman diagram (a) is the same as the sum of the two LF time-ordered diagrams (b) and (c), respectively.

The transition form factor $F_{P \gamma}$ for the $P \rightarrow \gamma^{*} \gamma\left(P=\pi^{0}, \eta, \eta^{\prime}\right)$ decay is defined from the matrix element of electromagnetic current $\Gamma^{\mu}=\left\langle\gamma(P-q)\left|J^{\mu}\right| P(P)\right\rangle=i e^{2} F_{P \gamma}\left(Q^{2}\right) \varepsilon^{\mu \nu \rho \sigma} P_{v} \varepsilon_{\rho} q_{\sigma}$, where $\varepsilon$ is the transverse polarization vector of the final (on-shell) photon. This process is illustrated by the Feynman diagram in Fig. 1 (a). For the LF calculation in parallel with the manifestly covariant one, we explored the two $q^{+} \neq 0$ frames which are defined in the timelike region, i.e. $\alpha=q^{+} / P^{+}=1-P^{\prime+} / P^{+}$frames with (1) $0<\alpha<1$ and (2) $\alpha=1$ cases. In the case of $0<\alpha<1$, the covariant diagram in Fig. 1 (a) is shown to be equivalent to the sum of two LF diagrams in Figs. 1 (b) and 1 (c). However, for the case of $\alpha=1$, we find that only Fig. 1 (c) contributes to the total transition amplitude and coincides with the the manifeslty covariant result. 
For instance, the LF result of $F_{\pi \gamma}\left(q^{2}\right)$ obtained from $\alpha=1$ limit in the exactly solvable BetheSalpeter (BS) model is given by [5]

$$
F_{\pi \gamma}\left(q^{2}\right)=\frac{\left(e_{u}^{2}-e_{d}^{2}\right)}{\sqrt{2}} \frac{N_{c}}{4 \pi^{3}} \int_{0}^{1} \frac{d x}{(1-x)^{2}} \int d^{2} \mathbf{k}_{\perp} \frac{m_{Q}}{M_{0}^{2}-q^{2}} \chi\left(x, \mathbf{k}_{\perp}\right),
$$

where $\chi\left(x, \mathbf{k}_{\perp}\right)=\frac{g}{x\left(M^{2}-M_{0}^{2}\right)}$ and $M_{0}^{2}=\frac{\mathbf{k}_{\perp}^{2}+m_{Q}^{2}}{x(1-x)}$ is the invariant mass. We also should note that our direct result of the form factor shows an excellent agreement with those obtained from the dispersion relations. This assured the reliability of our numerical calculation in the timelike region. We then mapped the BS model with the LF vertex function $\chi$ to the standard LFQM with the gaussian wave function $\phi_{R} \propto e^{-M_{0}^{2} / 8 \beta^{2}}$ following the same correspondence relation $\sqrt{2 N_{c}} \frac{\chi\left(x, \mathbf{k}_{\perp}\right)}{1-x} \rightarrow \frac{\phi_{R}\left(x, \mathbf{k}_{\perp}\right)}{\sqrt{\mathbf{k}_{\perp}^{2}+m_{Q}^{2}}}$ that we found in our previous analysis of two-point and three-point functions for the pseudoscalar and vector mesons [8]. This allowed us to apply the more phenomenologically accessible Gaussian wave function provided by the LFQM analysis of meson mass spectra. More detailed calculations can be found in [5].

For $\left(\eta, \eta^{\prime}\right) \rightarrow \gamma^{*} \gamma$ transitions, we take into account the presence of the strange quark and antiquark components in the $\eta$ and $\eta^{\prime}$ mesons as well as their mixing with the non-strange quark and antiquark components. To check the sensitivity of our LFQM, we use the mixing angle $\phi=$ $37^{\circ} \pm 5^{\circ}$ in the quark-flavor basis $\eta_{q}=(u \bar{u}+d \bar{d}) / \sqrt{2}$ and $\eta_{s}=s \bar{s}$ for our numerical calculations.

\section{Results}

In our numerical calculations within the standard LFQM, we use the model parameters obtained from the calculation of meson mass spectra using the variational principle in our LFQM [6, 7].
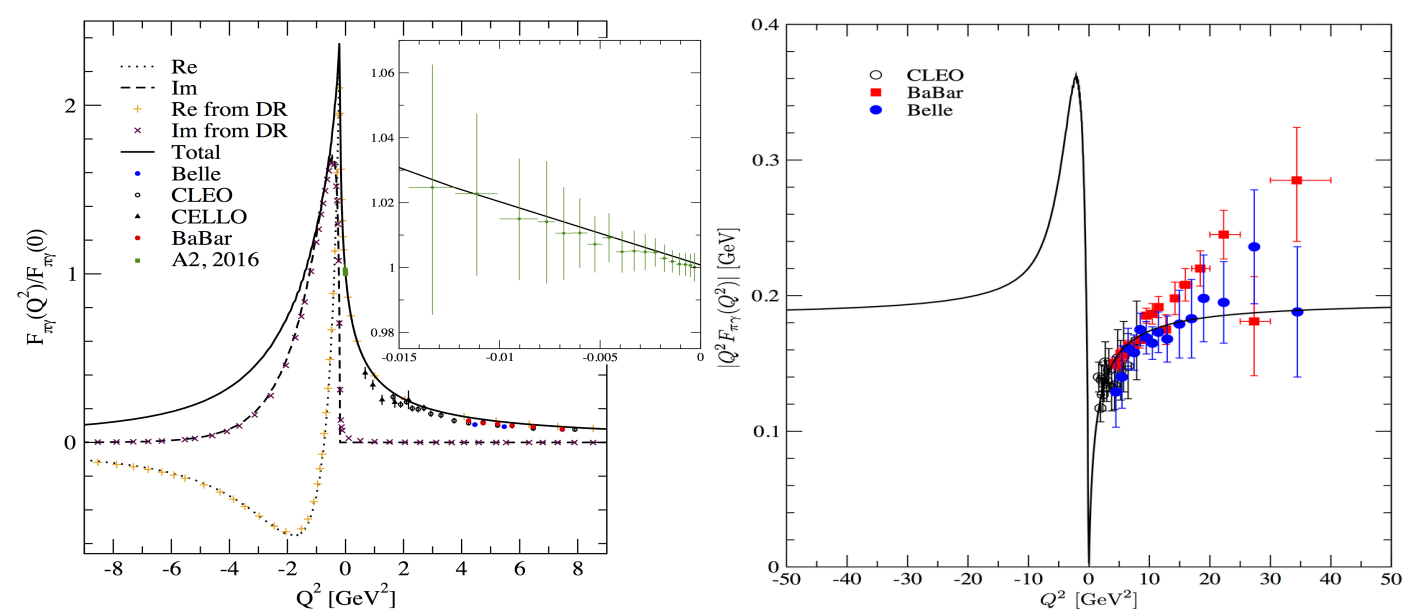

Figure 2: $F_{\pi \gamma}\left(Q^{2}\right) / F_{\pi \gamma}(0)$ (left panel) and $\left|Q^{2} F_{\pi \gamma}\left(Q^{2}\right)\right|$ (right panel) for both timelike $\left(q^{2}=-Q^{2}>0\right)$ and spacelike $\left(q^{2}=-Q^{2}<0\right)$ momentum transfer regions. The data are taken from $[3,4,9]$.

In Figs. 2 and 3, we show $F_{\left(\pi^{0}, \eta, \eta^{\prime}\right) \gamma}$ for both timelike and spacelike momentum transfer regions. For the low-energy regime, our results for the TFFs and their slope parameters are in good 

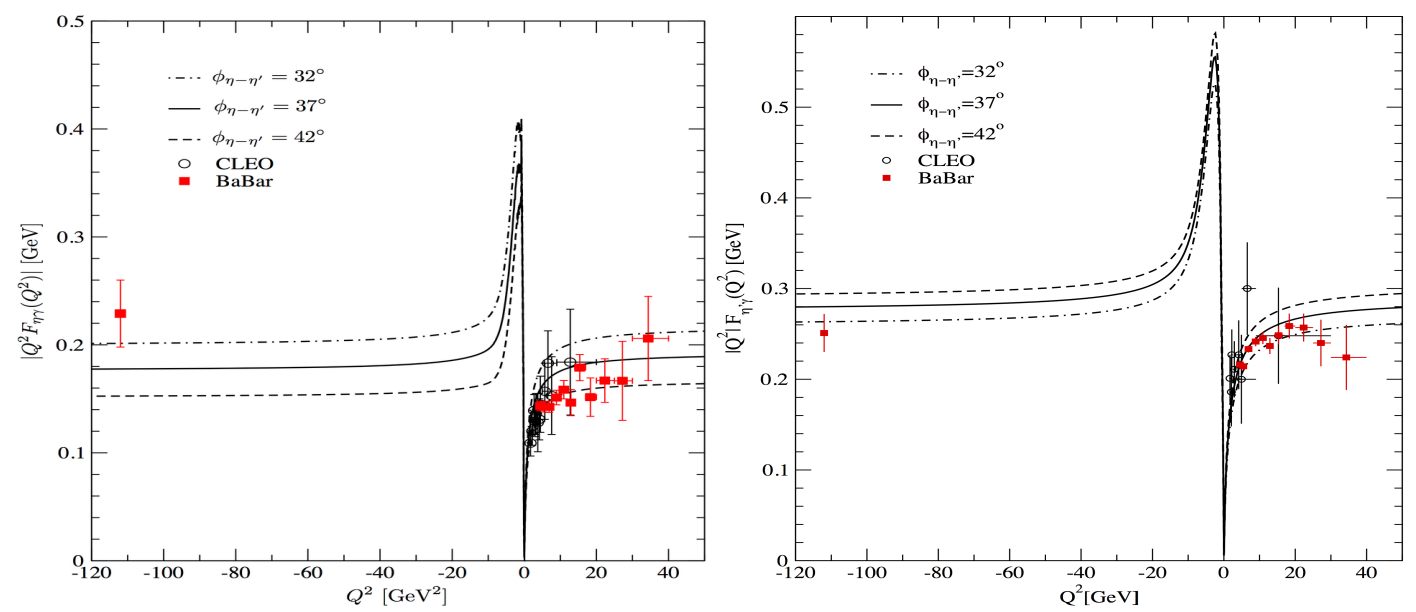

Figure 3: $\left|Q^{2} F_{\eta \gamma}\left(Q^{2}\right)\right|$ (left panel) and $\left|Q^{2} F_{\eta^{\prime} \gamma}\left(Q^{2}\right)\right|$ (right panel). The dot-dashed, solid, and dashed lines are results obtained from the mixing angles with $\phi_{\eta-\eta^{\prime}}=32^{\circ}, 37^{\circ}$ and $42^{\circ}$, respectively, and the data are taken from $[9,10]$.

agreement with the available data from the Dalitz decays of $\left(\pi^{0}, \eta, \eta^{\prime}\right)$ mesons. We also anticipate from our LFQM analysis that the experimental data for both timelike $F_{\eta \gamma}$ and $F_{\eta^{\prime} \gamma}$ would show peaks near $q^{2}=M_{\rho}^{2}$ (primary) and $q^{2}=M_{\phi}^{2}$ (secondary) corresponding to our primary and secondary peaks at $q^{2}=4 m_{u(d)}^{2}$ and $m_{s}^{2}$, respectively. For the high-energy regime, our result of $\left|Q^{2} F_{P \gamma}\left(Q^{2}\right)\right|$ does not show any steep rising behavior for high $\left|Q^{2}\right|$ region as measured from the BaBar Collaboration [3] but shows scaling behavior for high $\left|Q^{2}\right|$ consistent with the pQCD prediction.

\section{References}

[1] F. Jegerlehner and A. Nyffeler, Phys. Rep. 477 (2009) 1, arXiv:0902.3360 [hep-ph].

[2] G.P. Lepage and S.J. Brodsky, Phys. Rev. D 22 (1980) 2157.

[3] B. Aubert et al. (BABAR Collaboration), Phys. Rev. D 80 (2009) 052002, arXiv:0905.4778 [hep-ex] .

[4] S. Uehara et al. (Belle Collaboration), Phys. Rev. D 86 (2012) 092007, arXiv:1205.3249 [hep-ex].

[5] H.-M. Choi, H.-Y. Ryu and C.-R. Ji, Phys. Rev. D 96 (2017) 056008, arXiv:1708.00736 [hep-ph].

[6] H.-M. Choi and C.-R. Ji, Phys. Rev. D 75 (2007) 034019, hep-ph/0701177.

[7] H.-M. Choi and C.-R. Ji, Phys. Rev. D 59 (1999) 074015, hep-ph/9711450; Phys. Lett. B 460 (1999) 461, hep-ph/9903496.

[8] H.-M. Choi and C.-R. Ji, Phys. Rev. D 89 (2014) 033011, arXiv:1308.4455 [hep-ph]; Phys. Rev. D 91 (2015) 014018, arXiv:1412.2507 [hep-ph].

[9] H.-J. Behrend et al. (CELLO Collaboration), Z. Phys. C 49 (1991) 401; J. Gronberg et al. (CLEO Collaboration), Phys. Rev. D 57 (1998) 33, hep-ex/9707031; P. Adlarson et al. (A2 Collaboration), Phys. Rev. C 9522 (2017) 025202, arXiv:1611.04739 [hep-ex].

[10] P. del Amo Sanchez et al. (BABAR Collaboration), Phys. Rev. D 84 (2011) 052001, arXiv:1101.1142 [hep-ex]. 